\title{
ANALYSE D'UN MOTEUR ULTRASONORE PIÉZOÉLECTRIQUE À L'AIDE DE LA MODÉLISATION
}

\author{
F. CLAEYSSEN, R. LE LETTY, B. HAMONIC* et R. BOSSUT* \\ CEDRAT RECHERCHE, $10 \mathrm{Ch}$. du Pré Carré, ZIRST, F-38240 Meylan, France \\ *Institut Supérieur d'Electronique du Nord, Laboratoire d'Acoustique, 41 Bd Vauban, F-59046 Lille \\ cedex, France
}

\begin{abstract}
Ultrasonic motors use a frictional force produced by high frequency vibrations created by the piezoelectric effect of the stator, to drive the rotor. These devices have been widely studied especially in Japan. A stator of a piezoelectric ultrasonic rotating motor, which uses Lamb waves vibrations, has been modelled using the finite element code ATILA. It takes into account three dimensional piezoelectric effects and electrical excitations. ATILA permits the computation of the resonance frequency and the effective coupling coefficient of each vibrational mode, the electrical impedance and the elliptical displacement field. The governing electromechanical coupling has been determined. Consequently, the selection of active material and stator geometry may be optimized to provide an improved transduction efficiency.
\end{abstract}

\section{Introduction}

Les moteurs piézoélectriques ultrasonores sont mus par une vibration ultrasonore. Ils font l'objet depuis quelques années, d'une attention particulière à l'étranger (notamment au Japon, aux U.S.A. et en Allemagne), en raison de leurs caractéristiques intéressantes notamment un couple élevé à faible vitesse et à l'arrêt. De nombreuses architectures ont ainsi été proposées. Après avoir rapidement décrit le principe de fonctionnement et une classification de ces moteurs, on s'intéresse dans cet article à une modélisation du couplage électromécanique à l'aide du code de calcul par éléments finis ATILA, pour un moteur rotatif piézoélectrique existant. Les résultats de simulation sont discutés et permettent d'optimiser la géométrie du moteur ainsi que le choix du matériau piézoélectrique.

\section{Principe de fonctionnement et classification des moteurs}

Le principe de fonctionnement des moteurs ultrasonores (linéaires ou rotatifs) est le suivant : chaque point d'une pièce -appelée le stator- est animé d'un mouvement elliptique. Le rotor pressé contre l'élément vibrant se déplace sous l'action des forces de frottements (figure 1). Le mouvement elliptique se décompose en deux composantes de vibrations orthogonales suivant $\mathrm{x}$ et $\mathrm{y}$. Il est à noter que, suivant la forme de l'ellipse, la valeur de la vitesse du rotor peut varier. Ces vibrations elliptiques sont obtenues en excitant des modes de résonance particuliers du stator à l'aide de céramiques piézoélectriques. 


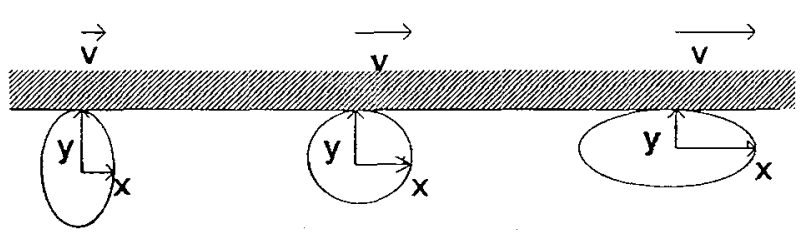

figure 1 : principe d'entraînement des moteurs ultrasonores : on considère que la force de frottement agit suivant $x$ et la force de contrôle de frottement, suivant $y$.

La conversion de l'énergie électrique en énergie mécanique peut être caractérisée dans un système par un coefficient dit de couplage effectif $\mathbf{k}_{\text {eff }}$. Ce coefficient dépend d'une part des caractéristiques piézoélectriques du matériau actif utilisé et d'autre part, de la géométrie du stator. II peut être calculé par la relation suivante :

$$
k_{\text {eff }}=1-f_{r}^{2} / f_{a}^{2} \text {, }
$$

où $f_{r}$ et $f_{a}$ sont respectivement les fréquences de résonance et d'antirésonance électrique.

Pour un matériau actif, trois types de couplage sont possibles :

- un couplage de type $\mathrm{k}_{33}$ correspond à une déformation suivant le même axe que l'excitation électrique,

- un couplage de type $k_{31}$ correspond à une déformation suivant un axe perpendiculaire à l'axe d'excitation électrique,

- un couplage de type $k_{15}$ correspond à un mouvement de torsion autour de l'axe d'excitation.

Les coefficients $k_{33}, k_{31}$ et $k_{15}$ sont les coefficients de couplage intrinsèque du matériau actif.

Pour optimiser les moteurs ultrasonores, il convient dans un premier temps d'identifier le type de couplage intervenant dans l'excitation des ondes ultrasonores du stator, et, dans un deuxième temps d'obtenir un coefficient de couplage effectif maximal s'approchant de la valeur du coefficient de couplage intrinsèque du matériau actif utilisé.

Il existe de nombreuses possibilités d'excitation pour aboutir à des déformations elliptiques, qui permettent de classer ces moteurs ultrasonores en deux grandes catégories [1] : les premiers utilisent un seul mode de vibration; les seconds en utilisent deux. Les moteurs monomodes utilisent soit une onde stationnaire, soit une onde progressive obtenue en superposant deux ondes stationnaires identiques avec un déphasage adéquat [2]. Les moteurs utilisant deux modes de vibrations utilisent, quant à eux, un couplage de vibrations. Si les deux modes sont excités indépendamment, et si les déplacements liés à chacun des modes sont orthogonaux, le moteur est parfois qualifié d'hybride [3]. Le moteur étudié ici est monomode et utilise une onde progressive de Lamb antisymétrique.

\section{Principe de la modélisation}

L'objet de l'étude est la modélisation du stator d'un moteur rotatif type USR45 (figure 2-a). Une telle modélisation réalisée à l'aide du code éléments finis ATILA [4], produit une image du comportement du moteur tenant compte de l'excitation électrique utilisée, des effets piézoélectriques tridimensionnels et des pertes dans les matériaux. Cette approche est a priori intéressante, puisque décrivant la conversion d'énergie électromécanique par un couplage fort, elle permet dans ce cas d'obtenir les résultats de calculs suivants :

- fréquences de résonance et d'antirésonance de chaque mode,

- coefficients de couplage effectif associés à chaque mode,

- courbe d'admittance électrique en fonction de la fréquence,

- amplitudes de vibrations (grand axe, petit axe, inclinaison de l'ellipse) en chaque point du stator et déformées d'ensemble de la structure. 

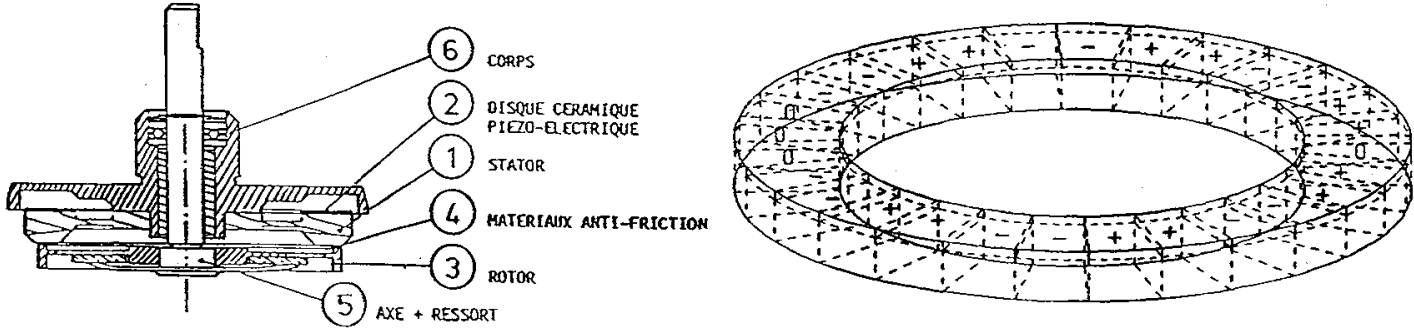

figure 2 : a) vue en coupe du moteur étudié (le stator est constitué des pièces 1 et 2 ), b) maillage du stator.

Le stator est constitué d'une couronne d'éléments piézoélectriques et d'un anneau métallique (figure 2-b). Les encoches présentes sur l'une des faces du stator ne sont pas modélisées ici pour limiter les temps de calcul. D'après le nombre de secteurs piézoélectriques, ce moteur fonctionne suivant un mode de flexion (parfois appelé mode de Lamb antisymétrique) tel que le nombre d'onde n sur la circonférence du stator soit égal, à la résonance, à 7 . La superposition de deux ondes stationnaires déphasées de $90^{\circ}$ permet de générer une onde progressive. Pour aboutir à cette superposition, les éléments piézoélectriques sont excités de la manière suivante :

- un premier groupe constitué de 12 éléments (ou 6 secteurs à polarisation altemée), est excité par une tension alternative de pulsation $\omega$ et de phase $0^{\circ}$,

- un second groupe également constitué de 12 éléments est excité par une tension alternative de pulsation $\omega$ et de phase $90^{\circ}$,

- ces deux groupes sont séparés d'un coté par 1 élément (soit 1/4 de longueur d'onde pour le mode de fonctionnement) et de l'autre par 3 éléments, tous 4 non excités.

Le calcul peut être effectué pour différents matériaux en tenant compte des pertes internes mécaniques et diélectriques, qui introduisent de l'amortissement.

\section{Résultats}

\subsection{Déformées de la structure au mode de flexion $n=7$}

Un premier calcul a été effectué en utilisant une céramique piézoélectrique de type LG144. La fréquence de résonance calculée du mode de flexion $\mathrm{n}=7$ est égale à $56.6 \mathrm{kHz}$ et est en accord avec un calcul analytique $(54.8 \mathrm{kHz})$. Le type de mode est identifié en visualisant la structure déformée à la résonance pour une phase de $0^{\circ}$ et de $90^{\circ}$ (figure 3). La différence entre les deux déformées se résume à un déplacement des crêtes de vibration, d'un quart de longueur d'onde. Elles permettent également d'identifier le sens de rotation du moteur. Une approche plus détaillée des déplacements montre que les amplitudes de vibration varient sensiblement (dans un rapport 3) suivant le rayon du stator, et que le déplacement a également une composante radiale, qui génère un frottement pur. Ces phénomènes ne sont pas sans conséquence sur le plan de l'usure du matériau antifriction assurant le contact entre le stator et le rotor et donc sur la longévité du moteur.
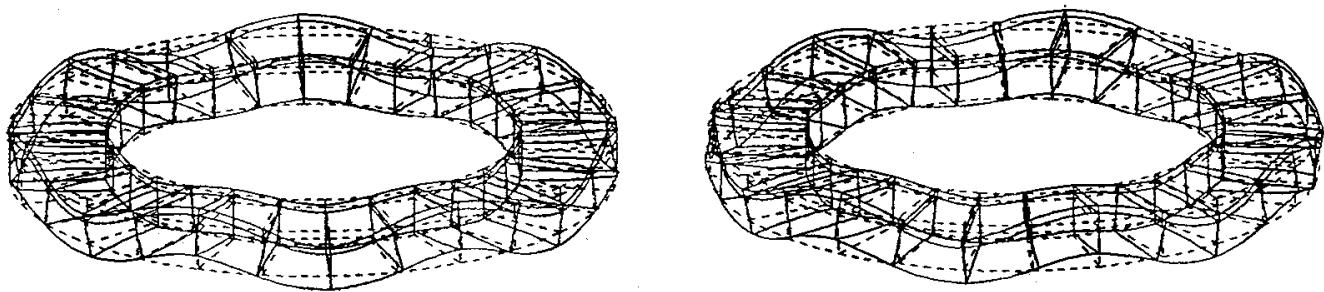

figure 3 : vue de la structure déformée excitée à la résonance aux instants de temps électrique $0^{\circ}$ et $90^{\circ}$ (la déformée est représentée en traits pleins et la structure au repos, en pointillés). 


\subsection{Admittance électrique}

L'admittance électrique du $1^{\mathrm{er}}$ groupe de céramiques est visualisée sur une bande de fréquence assez large (figure 4). Elle permet de constater l'existence de plusieurs fréquences de résonance couplées électriquement, qui correspondent respectivement aux modes de flexion $n=6,7$ et 8 . Les coefficients de couplage effectif associés à chaque mode est 9.6,11.0 et $9.1 \%$ en bon accord avec les valeurs expérimentales. Ce résultat de calcul montre que le fonctionnement d'un tel moteur est possible sur les trois modes, ce qui a été vérifié expérimentalement.

\subsection{Type de couplage mis en jeu}

Un second calcul a été effectué en prenant un matériau piézoélectrique présentant un coefficient piézoélectrique $d_{31}$ très petit. Pratiquement, un couplage de type $k_{31}$ ne peut être obtenu avec ce matériau.

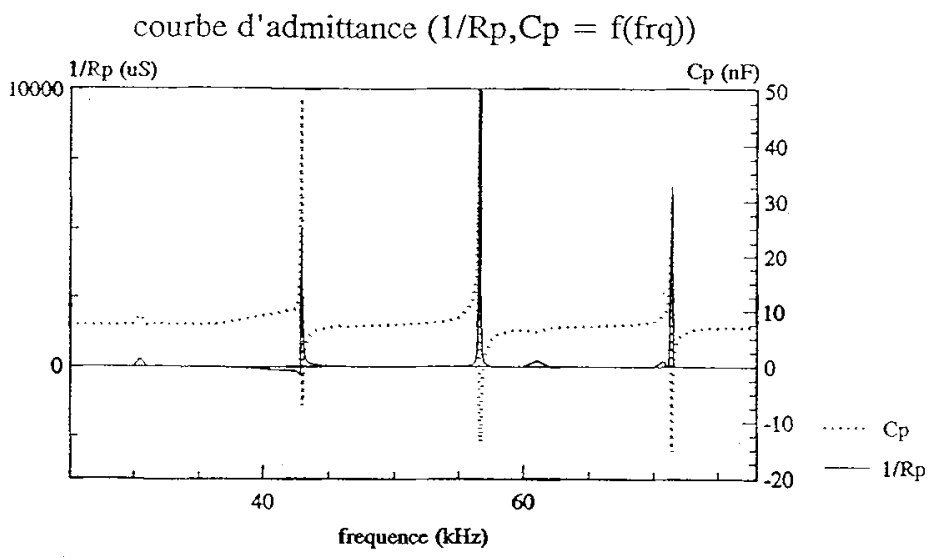

figure 4 : courbe d'admittance électrique calculée en fonction de la fréquence.

Dans ce cas, les valeurs du coefficient de couplage effectif calculé restent inférieures à $2 \%$. Par ailleurs, les déplacements à la résonance sont très faibles. On en déduit donc que le couplage électromécanique mis en jeu dans cette architecture de moteur est de type $\mathbf{k}_{31}$. Pratiquement, il signifie que la vibration en flexion du stator est obtenue par des allongements/contractions du matériau piézoélectrique suivant la circonférence du stator et non suivant la direction d'excitation électrique.

\section{Conclusion}

L'analyse du fonctionnement d'un moteur ultrasonore piézoélectrique type USR45 a été réalisée à l'aide d'une modélisation par la méthode des éléments finis avec le logiciel ATILA en prenant en compte les effets piézoélectriques et l'excitation électrique utilisée. La modélisation du stator a permis de retrouver les fréquences de résonance sur lesquelles le moteur fonctionne, les coefficients de couplage effectifs et les formes de déplacements elliptiques. Le type de couplage électromécanique mis en jeu a été identifié et l'importance du coefficient de couplage effectif comme critère d'analyse d'un moteur a été mise en évidence. Il permettra en particulier un choix judicieux du matériau piézoélectrique.

Avec les moyens de modélisation actuels, il est possible de la même façon d'optimiser les paramètres géométriques du stator de ce moteur. Plus généralement, l'intérêt de la modélisation permet d'envisager à présent l'analyse et la mise au point d'une grande variété de moteurs uiltrasonores. 


\section{Références}

[1] Y.Tomikawa, T.0gazawara, T.Takano, "Ultrasonic motors - Constructions / Characteristics / Applications", Ferroelectrics, vol. 91, pp 163-178, 1989.

[2] T.Takano, Y.Tomikawa, T.Ogazawara, S.Sugawara, M.Konno, "Ultrasonic motors using piezoelectric ceramic multimode vibrators", IEEE Ultr., Fer. and Freq. Control, vol. 37(2), pp 224$229,1990$.

[3] M.Kurosawa, S.Ueha, "Hybrid transducer type ultrasonic motor", IEEE Ultr., Fer. and Freq. Control, vol. 38(2), pp 89-92, 1991.

[4] J.N.Decarpigny, B.Hamonic, O.B.Wilson, "The design of low-frequency underwater acoustic projectors: Present status and future trends", IEEE Oceanic Eng., vol. 16(1), pp 107-122, 1991. 